\title{
Image du corps, idéal corporel et féminité hégémonique chez les enseignantes d'EPS
}

\section{Geneviève Cogérino et Marie Mansey}

\section{(2) OpenEdition}

\section{Journals}

Édition électronique

URL : https://journals.openedition.org/trema/1138

DOI : $10.4000 /$ trema. 1138

ISSN : 2107-0997

Éditeur

Faculté d'Éducation de l'université de Montpellier

\section{Édition imprimée}

Date de publication : 1 juin 2010

Pagination : 95-110

ISSN : 1167-315X

\section{Référence électronique}

Geneviève Cogérino et Marie Mansey, «Image du corps, idéal corporel et féminité hégémonique chez les enseignantes d'EPS », Tréma [En ligne], 32 | 2010, mis en ligne le 01 juin 2012, consulté le 16 février 2023. URL : http://journals.openedition.org/trema/1138; DOI : https://doi.org/10.4000/trema.1138

Ce document a été généré automatiquement le 16 février 2023.

Tous droits réservés 


\title{
Image du corps, idéal corporel et féminité hégémonique chez les enseignantes d'EPS
}

\author{
Geneviève Cogérino et Marie Mansey
}

1 L'éducation physique et sportive (EPS) en tant que discipline scolaire est devenue un objet d'intérêt pour les chercheurs s'intéressant aux problématiques de genre. Peu de travaux français sont relatifs au corps ou à l'image du corps de l'enseignant ou de l'élève. Or, la discipline EPS accorde une place centrale au corps.

2 Notre projet est d'étudier l'image du corps de l'enseignante d'EPS. PUJADE RENAUD (1983) a traité cette question dans une

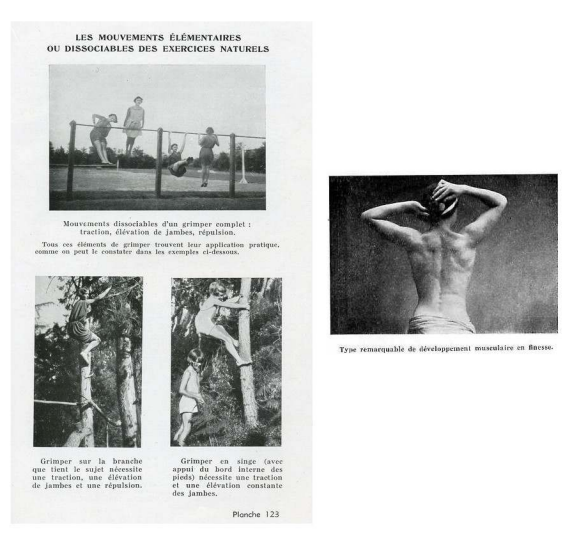
perspective psychanalytique. Elle a étudié les discours des enseignants et montré que le corps de l'enseignant dans la classe est une réalité ayant un impact non négligeable dans la relation pédagogique. Elle souligne que les femmes enseignantes se sentent inférieures aux hommes enseignants, enviant leur charisme, leur autorité naturelle, leur stature. L'auteur va jusqu'à envisager que la femme enseignante se sentirait fautive de ne pas être un homme. Elle émet l'hypothèse qu'il existerait chez ces dernières une volonté de mettre entre parenthèses un corps sexué pour cacher un manque. Qu'en est il alors des enseignantes d'EPS ? Quelle place accordent elles au corps féminin?

L'EPS dans les programmes nationaux se veut égalitariste, prônant la co-éducation et le respect de toutes les différences et en particulier des différences sexuées. Elle reste néanmoins une discipline qui utilise le sport par le biais des activités physiques et sportives (APS) comme moyen d'enseignement. Or, le sport est considéré comme un domaine porteur des valeurs associées à la masculinité socialement dominante. 
Quelle peut être la place de la féminité dans l'image que les enseignantes d'EPS ont de leur corps au sein de la relation pédagogique? Existe-t-il chez ces dernières des tensions $\mathrm{du}$ fait de l'origine masculine du sport? Ces questions constituent le point de départ d'une recherche, très partiellement présentée dans le cadre de cet article. La première partie précise ce que l'on entend par féminité et masculinité hégémoniques. La seconde distingue image du corps dans une perspective psychosociale et idéal corporel. La troisième contextualise les questions de recherche dans le domaine du sport ou de l'EPS.

\section{Féminité et masculinité : des constructions culturelles}

5 Si le terme « sexe » renvoie aux différences biologiques, le terme " genre » renvoie à « un réseau de représentations, établies sur les standards culturels en matière de masculinité et de féminité, spécifiant les droits et les devoirs ainsi que la manière d'être des deux groupes » (DURAND-DELVIGNE, 1990, 70). Le genre est une construction en interaction permanente avec des normes socio - culturelles. Les différences de comportements, de valeurs, d'attitudes, d'occupations mais aussi de rôles sociaux entre hommes et femmes sont la conséquence d'une socialisation différenciée selon le sexe, transmise par les parents, les pairs, les médias et toutes les instances éducatives. CUSUMANO et THOMPSON (1997) distinguent l'exposition aux normes, leur conscience et leur intériorisation. La féminité et la masculinité sont donc des repères culturels qui fixent les comportements, attitudes et valeurs attendus au sein d'un groupe social, mais qui peuvent être diversement appropriés selon les individus.

\section{1. Féminité et masculinité hégémoniques}

6 CONNELL (1995) envisage l'existence d'un gender order, un ordre social des genres, au sommet duquel domine la masculinité hégémonique. L'ordre des genres renvoie à un système dynamique de relations de pouvoir dans lequel de multiples masculinités et féminités sont constamment construites, contestées, modifiées. CONNELL situe la masculinité hégémonique comme «la conception sociale dominante, l'idéal culturel et l'idéologie sociale». Elle se différencie des masculinités subordonnée (en position dominée, voire même opprimée) et complice (masculinité qui sans être hégémonique se rapproche de celle-ci par divers ajustements). On reconnait parallèlement l'existence d'une forme de féminité culturellement idéalisée, complice de la masculinité hégémonique. CONNELL préfère le terme emphazised femininity alors que de plus en plus d'auteurs, à l'instar de KRANE (2001) adoptent « féminité hégémonique » pour désigner la forme culturellement dominante de la féminité.

\section{2. Attributs de la féminité et masculinité hégémoniques}

7 La féminité et la masculinité, constructions culturelles, définissent des attributs, des comportements, des valeurs, des attitudes, des occupations et rôles sociaux particuliers, socialement valorisés et attachés à l'un des deux sexes. La force, la ténacité, la compétence, le potentiel sexuel, l'indépendance, la dominance et l'agressivité constituent les attributs essentiels de la masculinité (comprise ici comme masculinité socialement valorisée). La féminité traditionnellement valorisée s'oppose aux attributs masculins: 
douceur, beauté, soumission, dépendance, repliement sur soi, finesse, souplesse en sont des attributs historiquement construits et constitutifs. Dans de cadre de la théorie socio culturelle, ces attributs sont pensés comme des normes auxquelles de nombreux individus tentent de se conformer.

\section{Féminité/masculinité hégémoniques et inscription corporelle}

8 D'un point de vue sociologique, le corps est lieu de mémoire, d'apprentissage d'habitudes culturellement construites et marqueur de positions sociales. En ce sens, le corps peut être considéré comme un lieu privilégié d'inscription des normes de genre. Il est possible de distinguer l'image corporelle que les femmes ont d'elles-mêmes, l'idéal corporel auquel elles se comparent et l'idéal corporel socialement admis.

\section{1. Idéal corporel féminin/masculin socialement valorisé}

Des idéaux corporels, spécifiques à un environnement social et culturel, définissent ce que doivent être corporellement les femmes ou les hommes pour être considérés comme féminines ou masculins. Le corps est envisagé ici comme l'ensemble des caractéristiques et attributs matériels visibles: particularités anatomiques (morphologie, couleur, taille des différents segments, visage, pilosité ...) et attributs extérieurs tels que vêtements, bijoux, maquillage.

10 D'une manière générale, le corps de la femme est un corps pensé comme un "objet esthétique " morcelé et soumis au regard: le nez, le visage, les hanches, les bras, la poitrine, les jambes, les pieds, les épaules, la coiffure ... (FREDRICKSON \& ROBERTS, 1997). À l'inverse, le corps de l'homme est pensé comme un corps «fonctionnel ». Il est caractérisé et évalué en fonction de sa force musculaire, son niveau d'énergie, son agilité, sa santé, sa condition physique, son degré coordination ou son activité sexuelle (FRANZOI, 1995). Cette conception qui distingue corps « objet esthétique » et corps «fonctionnel » est une conception dominante, révélatrice de la distinction entre l'idéal corporel féminin et l'idéal corporel masculin.

11 Le standard masculin met l'accent sur la force et le développement musculaire du corps. Actuellement, l'idéal morphologique masculin valorise une silhouette de type mésomorphique (en forme de " $\mathrm{V} »$ ) mettant ainsi en valeur des épaules larges, des hanches étroites, des abdominaux plats et nettement dessinés.

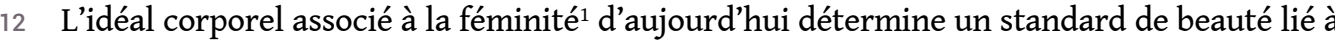
la minceur. Les études comparatives entre 1959 et 1988 ont mis en évidence une baisse des mesures corporelles (taille du buste, des hanches, poids) des modèles féminins photographiés pour les magazines (WISEMAN, GRAY, MOSIMANN \& AHRENSE, 1992). Cet idéal de minceur est perçu dès l'enfance. Avant 8 ans, les fillettes disent déjà avoir conscience de cet idéal (GROGAN \& WAINWRIGHT, 1996). Si socialement le corps féminin considéré comme normal est un corps mince, il est aussi un corps enjolivé par les bijoux, le maquillage et des vêtements mettant en valeur la silhouette. On en parle comme un corps fragile, faible, non énergétique, tendre, un corps passif, gracieux. Un corps aux antipodes de la force, du muscle ou de la puissance. 


\section{2. Définition de l'image du corps}

13 La psychologie sociale envisage l'image du corps comme un concept multidimensionnel. Celui-ci renvoie à «l'expérience psychologique à multiples facettes liée au fait d'être « incarné », expérience qui ne concerne pas seulement sa propre apparence [...]. Ce concept se réfère à des préoccupations, des croyances, des sensations, des comportements " (CASH, 2004). Il revêt deux dimensions: l'une affective et l'autre cognitive et attitudinale. Les auteurs distinguent alors une dimension évaluative de l'image du corps et une dimension investissement. La première renvoie aux croyances et évaluations individuelles que chacun porte sur son apparence physique, la seconde à l'importance accordée à la conformité, réelle ou supposée, à l'égard du standard social (MUTH \& CASH, 1997).

L'estime de soi globale de chaque individu est dépendante de la valeur de soi physique globale, elle-même dépendante de différentes perceptions que l'individu porte sur luimême: perceptions de sa compétence physique, de sa force, de sa souplesse, de sa coordination mais aussi de son apparence physique (FOX \& CORBIN, 1989). L'image que l'individu a de son corps, les perceptions qu'il porte sur ce dernier influencent donc l'estime qu'il a de lui-même.

\section{3. Idéal corporel associé à la féminité hégémonique et insatisfactions corporelles}

\section{3. 1. Comparaison, insatisfactions et conflits de genre}

Les études en psychologie sociale s'inscrivant dans la théorie socio - culturelle ont montré que la majorité des individus ont tendance à comparer l'image corporelle qu'ils ont d'euxmêmes à un idéal corporel construit à partir des idéaux socialement valorisés.

Dans de nombreux cas, cette comparaison est à l'origine d'insatisfactions corporelles, source de stress, culpabilité, dépressions et parfois désordres alimentaires. Les deux sexes sont touchés par cette problématique, mais les femmes sont davantage insatisfaites par leur image corporelle que les hommes (TAYLOR \& COOPER, 1986). Elles accordent une importance à la finesse de leur silhouette et se pensent plus corpulentes qu'elles ne le sont réellement. Enfin si l'insatisfaction face à l'image du corps reste constante quel que soit l'âge de la femme, l'investissement dans des stratégies visant à modifier cette image diminue au cours du temps.

17 En intégrant des valeurs, des comportements, des attributs corporels liés à l'activité sportive pratiquée, les femmes intègrent aussi des valeurs, comportements et attributs connotés comme masculins. Elles peuvent alors avoir des difficultés à se positionner identitairement, faisant émerger un conflit de genre mais il semble qu'il n'existerait pas plus de conflit de genre chez les femmes sportives que chez les femmes non-sportives (DESERTRAIN \& WEISS, 1988 ; MILLER \& LEVY, 1996).

\section{3. 2. Stratégies visant à concilier image corporelle et idéal corporel}

18 Chez les hommes comme chez les femmes, des stratégies permettent de concilier image corporelle et idéal corporel. WIENKE (1998) identifie trois stratégies chez les hommes. Dans un premier cas les hommes tentent de s'approcher au plus près des caractéristiques 
du corps idéal reconnu socialement : «la stratégie de dépendance ». Ils travaillent donc leur corps pour revêtir une apparence idéalement valorisée, ceci peut passer par des régimes, de l'activité physique pour prendre du muscle, un choix approprié de vêtements. Ici le corps est envisagé comme un objet fonctionnel et l'idéal corporel socialement valorisé est entendu comme un moyen de se faire accepter par les autres. Dans le cas de la "stratégie de reformulation", les hommes tentent d'ajuster les standards de la masculinité hégémonique afin que ceux-ci se coordonnent à leur propre image corporelle. La «stratégie de rejet » se concrétise par un rejet actif du modèle masculin dominant et la construction d'une identité masculine alternative. Cette étude, exclusivement centrée sur une population masculine, peut être mise en parallèle avec la formalisation d'USSHER (1997) qui émet l'idée que les femmes selon les situations et les contextes dans lesquels elles évoluent, choisissent de "faire femme ", «d'être femme» ou de "résister à la femme ».

\section{Sport, EPS et féminité}

\section{1. Le sport, territoire masculin}

19 Le sport est un lieu de préservation de la masculinité (THEBERGE, 1985). Les valeurs sportives (compétition, autonomie, indépendance, force, puissance, performance) mettent en avant les valeurs de la masculinité hégémonique précédemment développées (MESSNER, 1988).

20 La performance sportive nécessite que les femmes aient un corps fort, puissant, musclé. Mais en intégrant ces attributs, ces dernières sont poussées à devenir inadaptées à l'idéal corporel féminin socialement valorisé (MESSNER, 1988; GREENLEAF, 2002; ROSS \& SHINEW, 2008). Ainsi les sportives sont face à une contradiction : être performantes et développer des attributs masculins (comme la musculature) tout en ne s'éloignant pas excessivement de l'idéal corporel féminin (KRANE et al. 2004).

21 Ces jeunes femmes sont conscientes que s'éloigner de cet idéal peut engendrer des conséquences négatives (mal-être) et stigmatisations diverses. Elles sont donc amenées à distinguer corps "athlétique » et corps "social». Certaines vont jusqu'à penser que lorsqu'elles pratiquent, elles ne sont pas femmes mais sont seulement des machines (KRANE et al. 2004). Si les femmes sportives ont une image positive de leur corps (MILLER \& LEVY, 1996) et apprécient leur corps athlétique, la puissance et la force qu'il dégage, a contrario elles ont des affects négatifs envers leur corps «social ». Plusieurs stratégies sont alors utilisées pour négocier l'écart existant entre l'image de leur corps et l'idéal corporel féminin. L'une d'entre elles consiste à renforcer sa féminité en dehors ou à l'intérieur du contexte sportif en valorisant les attributs associés à la féminité hégémonique : maquillage, vêtements, coiffures et alimentation appropriée (KRANE et al. 2004). Les femmes sportives entrent dans une féminité complice à la féminité hégémonique, elles tentent de « faire femme » lorsqu'elles se donnent à voir (KRANE et al. 2004 ; ROSS \& SHINEW, 2008). D'autres sportives, à l'inverse, tentent de repousser les limites de la féminité hégémonique et donc " résister à la femme ».

Les activités sportives sont des « territoires sexués » : le rugby, le foot, la boxe, le cyclisme sont considérés comme des activités sportives masculines alors que la danse, la gymnastique, le patinage sur glace sont envisagés comme des activités spécifiquement féminines. Cette distinction perçue dès l'adolescence (FONTAYNE, SARRAZIN \& FAMOSE, 
2001) est porteuse d'un rapport différent au corps féminin. KRANE et al. (2004) montre qu'en général les joueuses de tennis (activité connotée comme "féminine») sont davantage concernées par le portrait traditionnel de l'apparence féminine alors que les joueuses de rugby ou hockey (activités connotées comme "masculines») tentent de repousser les limites de la féminité.

\section{2. Quelles implications chez l'enseignante d'EPS ?}

L'EPS en tant que discipline scolaire utilise aujourd'hui les activités sportives comme un moyen au service d'objectifs éducatifs plus larges. Bien que ne se confondant pas avec les activités sportives, elle reste néanmoins un domaine proche de ces dernières. Les enseignants hommes ou femmes sortant actuellement des formations STAPS sont issus de cursus sportifs. Il est alors intéressant d'étudier les liens entre sport et féminité chez les enseignantes d'EPS.

Les femmes enseignantes sont soumises comme bien d'autres à l'idéal corporel féminin socialement valorisé. Mais pour être crédible face à ses élèves, l'enseignante d'EPS doit adopter une apparence sportive. Un enseignant d'EPS d'apparence physique "enrobée " est perçu comme moins compétent par des lycéens et suscite un attrait moindre pour la discipline (MELVILLE \& MADDALOZZO, 1988).

Quelle image ces enseignantes ont-elles alors de leur corps au sein de la relation pédagogique? Quelle place accordent-elles à l'idéal corporel féminin socialement valorisé : quelles normes corporelles valorisent-elles dans le domaine de l'EPS et en dehors? Vivent-elles la contradiction que vivent les femmes sportives qui différencient leur « corps athlétique » de leur « corps social»?

\section{Méthodologie}

Onze enseignantes d'EPS ont été interviewées : huit enseignent en collège et trois en lycée. Bien que relevé, l'âge, variant de 26 à 53 ans, ne constitue pas une variable prise en compte étant donné la taille de l'échantillon. Leurs spécialités sportives sont diverses et reflètent la distinction entre activités sportives connotées féminines, masculines ou neutres: handball, basket-ball, danse, gymnastique, athlétisme, natation, volley ball. Toutes sauf deux ont pratiqué leur spécialité au niveau régional ou national; leur investissement dans cette pratique varie de 7 à 25 ans.

L'entretien semi - directif est le plus approprié pour cette approche qualitative et exploratoire. Le guide d'entretien recense les thèmes et sous - thèmes à aborder.

- - Image du corps au sein de la relation pédagogique

Le but est de caractériser l'image du corps des enseignantes d'EPS, de situer l'importance qui lui est accordée, ainsi que le modèle socioculturel de genre auquel elle se rapporte. Il s'agit d'amener les enseignantes d'EPS à parler de leur corps en rapport avec la féminité, durant le cours d'EPS et au sein de la relation pédagogique (ressentis, rapport aux élèves, importance accordée aux vêtements, maquillage,... importance accordée à l'apparence sportive, caractéristiques physiques, voix, place de la féminité).

- - Idéal corporel de l'enseignante d'EPS 


$$
\text { chis }
$$
tenue vestimentaire, aux apparences extérieures hors du contexte de l'EPS. Le but est de
déterminer un possible écart entre corps «enseignant » et corps «social » (en réunion Il s'agit de s'intéresser aux ressentis des femmes vis à vis de leur corps, de les amener à parents / professeurs, en conseil de classe et même en dehors de la sphère éducative),

- - Idéal corporel féminin

31 Le but est de comprendre comment les femmes envisagent la féminité, l'idéal corporel féminin, la place qu'elles leur accordent dans leur vie, d'identifier leur positionnement face à l'idéal corporel féminin socialement reconnu dans l'enseignement de l'EPS et en dehors.

La durée moyenne des entretiens est de $62 \mathrm{~min}$. La retranscription constitue un corpus de 66477 mots. L'anonymat des propos tenus lors de l'entretien et le droit de regard sur la retranscription ont été garantis.

À la suite d'une lecture flottante de l'ensemble du corpus, des thèmes et sous-thèmes ont été repérés. Les entretiens ont ensuite été découpés en énoncés et classés dans les rubriques correspondantes.

\section{Résultats}

Les résultats abordent l'importance que les enseignantes d'EPS accordent au corps au sein de l'EPS, puis la manière dont elles conçoivent leur propre corps ainsi que le corps idéal. L'écart avec les normes de genre socialement valorisées ainsi pointé permettra de définir la place accordée aux normes de la féminité hégémonique. Rappelons que nous entendons par image du corps, les préoccupations, les croyances, les sensations, les comportements qu'un individu peut avoir vis-à-vis de son corps (Cash, 2004).

\section{1. Importance de l'image du corps dans l'enseignement de l'EPS}

Les enseignantes d'EPS reconnaissent une importance primordiale au corps dans leur métier, mais relativisent cet aspect, et considèrent que l'enseignement de l'EPS est une profession de représentation.

\section{1. 1. L'image donnée à voir de son corps : une composante du métier reconnue par toutes.}

Toutes les enseignantes d'EPS interrogées reconnaissent que le corps ou l'image qu'elles donnent à voir par leur corps tient une place importante dans leur métier, quelles que soient leurs spécialités sportives d'origine ou leur milieu d'enseignement. L'enseignante d'EPS se trouve être au centre de la scène pédagogique : «On est directement sous les projecteurs [...] On se donne vraiment à voir » (E7). 

gestes, à leurs tenues vestimentaires comme à leur apparence. «Ils sont attentifs, ouais, attentifs à la façon dont tu es vêtue et à la façon dont tu bouges ton corps. Ils sont très attentifs à ça. » (E5)

L'image qu'elles donnent à voir aurait une influence directe sur le contact avec les élèves, leur engagement, leur motivation et leur écoute. Les caractéristiques corporelles sont pensées comme des moyens permettant d'orienter les comportements des élèves. Car ce sont bien ceux-ci, plus que les collègues ou les personnels administratifs, qui sont au centre de leurs préoccupations.

Les enseignantes tentent dans un second temps de relativiser ces propos communs à l'ensemble des interviewées. Deux stratégies sont alors utilisées. La première conduit à affirmer qu'elles ne portent aucune attention particulière à l'apparence corporelle. La seconde consiste à affirmer fortement que l'enseignement ne peut se réduire à l'image corporelle de l'enseignante.

\section{1. 2. Deux stratégies pour relativiser l'importance de l'image du corps}

Six des enseignantes interrogées affirment que l'attention qu'elles portent à leur corps et leur apparence n'est pas primordiale. Tout en reconnaissant l'importance de l'apparence, elles affirment dans un second temps ne pas y attacher une attention particulière, mais font part de leur volonté de "rester dans le coup", d'utiliser des formes de vêtements modernes, de ne pas trop grossir ou encore de faire des efforts afin de rester coquette : «Je ne vais pas enfiler mes vieux bas de survêt' classiques euhh... tu remplis pas les fesses parce que c'est coupé pour les hommes, ça flotte au niveau de ... où tu vois tu ressembles plus à un sac à patates [...]. Moi je pense que je suis coquette, je fais des efforts pour » (E4).

41 Sept enseignantes soutiennent dans un second temps que l'image ou l'apparence corporelle qu'elles donnent à voir ne constituent en rien le "fond de la question ». L'image qu'elles dégagent est à prendre en compte mais celle-ci ne peut être considérée comme la pierre angulaire de l'enseignement. «La crédibilité se fait au sein du cours, avec les situations, les transformations et le contact avec les élèves » (E6).

42 Ainsi, les données révèlent une certaine ambiguïté face à l'importance accordée à ce corps et l'attention portée à ce dernier. Comme si le paraître n'était qu'une activité superficielle, les enseignantes rechignent à accepter l'attention qu'elles lui accordent. Car reconnaître qu'elles portent attention à leur apparence les engage à avouer aux autres, et s'avouer à elles mêmes, leur soumission aux normes extérieures et leur appartenance à un monde de « superficialité ».

\section{2. Image que les enseignantes d'EPS ont de leur corps en EPS}

43 Nous présenterons successivement les caractéristiques communes à l'ensemble des enseignantes interrogées, leurs insatisfactions, leurs divergences à propos de la place accordée aux normes corporelles associées à la féminité hégémonique.

\section{2. 1. Un corps fonctionnel, un corps sportif}

Quels que soient leur spécialité sportive et le niveau sportif auquel elles ont évolué ou leur ancienneté, les enseignantes interrogées pensent leur corps de manière 
fonctionnelle (FRANZOI, 1995). Elles évaluent leur corps non pas en fonction de la perception qu'elles ont de différentes parties de ce dernier mais davantage en fonction des perceptions qu'elles ont de leur condition physique, de leur force musculaire, de leur niveau d'énergie ou d'agilité. Le corps qu'elles pensent mettre en avant en EPS est avant tout un corps actif, dynamique, tonique, capable de faire. Plus qu'un objet esthétique, ce corps est un moyen de démontrer, de participer avec les élèves, de les aider ou de les dynamiser : «J'ai l'impression que pour mes élèves l'image que je leur donne c'est l'image du corps d'une femme qui peut faire beaucoup de chose avec son corps» (E5) ; « Moi, je participe avec les élèves [...] Je me mets à plat ventre avec les élèves. Tu peux arriver et me trouver à plat ventre dans la classe. Je suis avec eux, je danse, je fais avec eux [...]. Je suis jamais assise sur le bord du terrain. Je ne veux pas donner l'image d'une mémère » (E1).

45 Cette fonctionnalité, cette tonicité, ce dynamisme sont gages de crédibilité dans leur métier et du respect des élèves: "Je fais, ouais [...] Je me mets moi aussi en jeu directement, quoi. Parce que tu vois, je leur demande de faire, de mettre en jeu leur corps et je trouve que, ouais... euh... c'est important aussi qu'ils voient que je suis capable de faire » (E7).

Plus encore qu'un corps actif, les enseignantes envisagent leur corps en EPS comme un corps à l'apparence sculptée par la pratique sportive. Selon les activités pratiquées, les enseignantes se disent "carrées", musclées au niveau des bras ou des mollets. Leur apparence sportive est une garantie de bien-être, moyen pour certaines de garder confiance face aux élèves et d'imposer le respect ou un rapport de force particulier : «Moi, je suis persuadée que le fait d'en imposer un peu physiquement parce que ... on les aide [les élèves], on les porte, on les ... Ils voient que même en tant que femme on a une certaine force, et bien, ça les... ça impose un respect » (E11). La carrure, moyen de se rassurer vis-à-vis de sa compétence à enseigner l'EPS, constitue un gage de crédibilité : «Mais tu vois mes épaules [un peu larges] bon ça ne me dérange pas. A la rigueur j'aime bien parce que ça va avec l'image du prof d'EPS, une image de sportive ». Au-delà, c'est la difficulté à être femme dans un milieu à forte connotation masculine qui se dévoile: «Moi je donne l'image d'un corps vivifiant, d'un corps fort [...] je veux montrer que la femme n'est pas forcément faible, donc pour moi c'est important » (E10).

Le sentiment de se sentir forte, capable d'en imposer, est évoqué par neuf des onze enseignantes, Quelles que soient leur spécialité sportive et leur morphologie : « C'est vrai que moi je fais $1 \mathrm{~m} 57$ et je ne serai pas fière d'arriver au LEP du bâtiment. Et en même temps je me sens forte. Je crois que je véhicule quand même une certaine puissance même si je n'ai pas une taille très, très... » (E6).

Les enseignantes sont donc conscientes du regard que les autres portent sur elles et des normes qu'ils imposent. Elles reconnaissent que l'image que chacun se fait de l'enseignante d'EPS est celle d'une femme au corps travaillé, sportif, musclé, tonique, capable de faire et de bouger. Etre en cohérence avec les représentations sociales partagées est essentiel mais le regard des élèves a ici plus d'importance que celui des collègues, de l'administration ou des parents d'élèves. Dans ces conditions, présenter certaines caractéristiques corporelles (telles que avoir des bras musclés ou une carrure imposante) n'est pas source d'insatisfaction. 


\section{2. 2. Les insatisfactions des enseignantes d'EPS} n'accorder aucune importance à la féminité en EPS telle qu'elle est corporellement et socialement définie. Elles sont issues de spécialités sportives connotées masculines (handball, basket ball). Pour elles, l'important dans ce domaine n'est pas de se «montrer femme », mais de se montrer "professeur d'EPS » : «Moi je suis prof d'EPS avant d'être une femme » (E9) ; « Non je ne suis pas hyper coquette parce que ce n'est pas mon style et puis je trouve qu'en EPS on n'en a pas besoin » (E7). attributs extérieurs (tenue vestimentaire) ne sont compris que dans cette perspective pragmatique. Plus que mettre en valeur le corps de la femme, il s'agit d'entrer en cohérence avec les besoins de la discipline : permettre au corps de se mouvoir de manière 
efficace. Si ces enseignantes d'EPS reconnaissent des limites de décence à ne pas franchir, les normes esthétiques associées à la féminité (mise en valeur du corps par les vêtements, maquillage, bijoux) n'ont aucun sens en EP pour elles : «Ben je fais attention qu'il n'y ait pas une tache sur mon pull ou un trou à mon pantalon [...] Mais moi je n'ai pas besoin de ça. Non, moi je suis vraiment dans le pratique, le fonctionnel, je ne veux pas perdre de temps avec ça [...] Ouais, je fais garçon manqué, c'est sûr » (E9).

Elles reconnaissent que les sportives, tout comme elles-mêmes, ne sont pas des modèles de féminité, et affirment ne pas correspondre aux normes corporelles de la féminité hégémonique. L'intégration mentale de ces normes corporelles reste toutefois effective. La féminité est associée au maquillage, aux bijoux, à des traits fins et des proportions fines, à des vêtements aux coupes et couleurs particulières. Mais ces enseignantes font le choix de ne pas mettre en avant ces aspects en EPS. Si ces femmes ne se reconnaissent pas corporellement comme féminines, elles affirment néanmoins se sentir féminines par d'autres aspects que l'apparence corporelle: le dialogue, la relation affective et maternelle avec les élèves.

Contrairement à ces quatre enseignantes, sept autres énoncent leur volonté et leur besoin de «se montrer femme» en EPS. La féminité comme précédemment est associée à la coquetterie, à des vêtements particuliers, au maquillage, aux bijoux. Elles ont pour volonté qu'on ne les reconnaisse pas seulement comme des enseignantes d'EPS mais aussi et surtout comme des femmes, en particulier grâce à leur tenue vestimentaire. Elles regrettent la neutralité de la tenue du "prof d'EPS». Sa fonctionnalité et son pragmatisme sont reconnus comme indispensables, ces tenues sont aussi envisagées comme des tenues non féminines, voir neutres : «On a une tenue qui est assez neutre, qui n'est pas connotée femme [...] C'est tout de même des tenues qui ne mettent pas en valeur la femme » (E8) ; « Moi je ne mets plus de survêtement. J'essaie de trouver des trucs euh... tu vois c'est pas un vrai survêt'. C'est plutôt des corsaires, des petits trucs, entre ville et tenues sportives. Je trouve qu'il y a un côté coquetterie » (E11); «On fait attention aux couleurs, à la forme, euh ...» (E2); «Alors c'est vrai que moi j'aime bien, je change de boucles d'oreille... j'essaye de cultiver un peu ce côté féminin quoi » (E8).

Le besoin de "se montrer femme» en EPS revêt différents intérêts. Tout d'abord, l'intégration par les femmes d'une partie des normes associées à la féminité hégémonique est considérée comme un facteur de bien-être. Mais les regards des élèves et des collègues jouent également un rôle. La conformité aux caractéristiques de la féminité est pensée comme un élément favorisant le contact et les relations positives entre individus. Enfin, le besoin de bouleverser le regard que l'on porte généralement sur les enseignantes d'EPS constitue un autre argument. Les femmes interrogées reconnaissent que les personnes environnantes ont des difficultés à voir l'enseignante d'EPS comme une femme.

Les enseignantes elles-mêmes se souviennent de leur propres enseignantes d'EPS, qui se montrant très sportives, niaient ou oubliaient de mettre en valeur ce côté femme : «On a tout de même eu beaucoup de profs hyper sportives toujours en jogging, pas maquillées, cheveux courts, un peu rondelettes. Enfin moi-même j'ai eu des profs comme ça. Et, si tu veux, je me dis qu'aux yeux des élèves euh ... J'avais pas envie de véhiculer ça » (E5).

Les femmes s'inscrivant dans cette perspective sont issues de spécialités sportives connotées féminines ou neutres. Elles ont par ailleurs une conception de l'acte d'enseignement qui met la séduction à la base de toute volonté d'enseigner: "Je pense qu'en tant que prof, on est sans arrêt en train de séduire [...] Je suis persuadée que c'est bien aussi qu'en tant que femme de véhiculer ... ce côté femme. » (E6). 


\section{3. Le corps idéal de la femme en EPS et en dehors de l'EPS}

61 L'objectif est de comparer les normes corporelles admises par les enseignantes en dehors de l'EPS (corps « social ») et au sein de l'EPS (corps « enseignant»).

62 Sept sur les onze sont réticentes à l'idée de reconnaître l'existence d'idéaux corporels dans le domaine de l'EPS ou à l'extérieur. Lorsqu'elles acceptent d'envisager un idéal, les enseignantes parlent davantage d'attitudes ou de valeurs que de caractéristiques corporelles. L'enseignante d'EPS idéale devrait être juste, ferme mais douce, proche de ses élèves et capable de leur proposer des contenus adaptés. La femme idéale devrait être une femme bien dans son corps, indépendante, capable de s'affirmer professionnellement et familialement.

\section{3. 1 Le corps idéal en dehors de l'EPS}

63 Lorsqu'elles tentent de définir le corps idéal de la femme, la minceur est la première caractéristique mise en avant: «Je dis qu'il n'y a pas d'idéal mais en même temps je trouve plus esthétique une femme fine qu'une femme obèse » (E7).

Elles mentionnent une taille dessinée, une poitrine marquée et une féminité assumée. Si cette féminité renvoie à des éléments tels que la douceur, le dialogue, la maternité, les enseignantes réaffirment le rôle de l'apparence. Féminité rime avec vêtements particuliers (jupes, décolletés, talons), cheveux longs, maquillage, bijoux, sourire, silhouette. Toutes ces caractéristiques font référence aux normes de la féminité hégémonique associées à la femme occidentale, blanche, hétérosexuelle. Les enseignantes en dehors de l'EPS sont donc en partie soumises à cet idéal, mais restent conscientes que celui-ci, relayé par les magazines et les différents médias, est une idéalisation de la femme qui ne peut être atteint. Elles n'hésitent pas à remettre en question certaines caractéristiques associées à cet idéal, comme l'image de la femme faible et passive.

Dans cette perspective, le discours sur le muscle prend un sens particulier. Le développement musculaire est apprécié par les enseignantes d'EPS, s'il n'est pas imposant. La finesse en est le critère majeur. Car une musculature imposante renvoie à l'idée de puissance et de masculinité. La sportive est donc valorisée dans la mesure où sa musculature lui permet de rester fine et «belle». Certaines sportives sont davantage évoquées: les "grimpeuses", les "danseuses», les "patineuses» ou les "GR». Les femmes sportives valorisées par leur silhouette sont donc celles pratiquant des activités connotées comme féminines. Le sport en dehors des activités "féminines » est pensé comme un domaine masculinisant les femmes.

66 Ces caractéristiques constitutives de l'idéal corporel féminin des enseignantes d'EPS hors du contexte de l'EPS ne valent pas forcément pour le domaine de l'EPS.

\section{3. 2. Le corps idéal de l'enseignante en EPS}

67 Sur de nombreux points, les onze femmes interrogées sont d'accord. Leur idéal est proche de l'image qu'elles ont de leur corps en EPS: un corps dynamique, tonique et «fonctionnel ». Ces caractéristiques permettent de favoriser l'engagement des élèves, de correspondre à l'image qu'ils en ont, d'être ainsi davantage crédible vis-à-vis de ces derniers et de se faire plus facilement respecter pour «tenir la classe » : «Je le vois bien 
les jours ou je n'ai pas l'énergie, on peut pas les laisser faire tous seuls. Si on est pas derrière pour impulser, pour relancer, pour les canaliser » (E9).

Le dynamisme, la tonicité et la fonctionnalité du corps sont mises en avant par les enseignantes, avec un corps jeune et svelte : « Si tu as une prof d'EPS un peu forte en face de toi, bon ben, ça ne va pas vraiment ensemble » (E9). Vieillesse et embonpoint ne sont pas des caractéristiques à associer au corps de la femme enseignante d'EPS (Webb \& Macdonald, 2007).

La voix forte, les épaules carrées, une musculature visible, une stature qui en impose et un corps fort sont valorisés et ce d'autant plus que les enseignantes envisagent des milieux difficiles : "C'est vrai que je pense que si tu es un peu plus large, t'as une carrure qu'en impose un peu plus je pense que ça peut ... tu vois, mettre en jeu un autre rapport de force » (E7); « Si on a plus de corpulence, je pense que les élèves ... ce n'est pas qu'ils respectent plus... mais ça impose un autre rapport » (E11).

Ainsi, selon le contexte (en ou en dehors de l'EPS), les enseignantes font part d'un changement de point de vue à propos de certains caractéristiques corporelles, ce qui montre bien que deux types de corps idéal sont distingués. Les épaules larges, les « gros bras » ou la carrure imposante appréciés en EPS, sont reconnus comme n'entrant pas dans les normes corporelles socialement admises: «Ben, moi j’ai quand même des gros bras [...] En EPS, non, non ça ne me dérange pas, parce que c'est plus cohérent avec le lieu. À l'extérieur, ouais, je me trouve trop musclée » (E6); «Je suis bien dans mon corps mais c'est vrai que un peu plus grande et un peu moins musclée pour l'extérieur ça pourrait être bien» (E7). La voix forte et le manque de poitrine sont des éléments dont la possession est diversement appréciée en fonction du contexte : les insatisfactions y sont attachées pour la vie «à l'extérieur », tandis que dans le cadre de l'enseignement de l'EPS aucune insatisfaction n'y est associée et mentionnée.

\section{Conclusion}

71 Le nombre d'entretiens réalisés constitue une limite à la généralisation des résultats. La poursuite des entretiens pourrait nous permettre de préciser les résultats en fonction de diverses variables : âge des enseignantes, milieu d'enseignement (ZEP avec de nombreux élèves d'origine maghrébine; établissements professionnels avec de nombreux garçons âgés) Nous pourrions ainsi cerner l'influence de ces contextes sur la manière dont les enseignantes envisagent leur corps en EPS et en dehors, ainsi que les idéaux corporels associés à la femme ou l'enseignante d'EPS. L'étude portant sur les représentations que les élèves ont de l'enseignante d'EPS selon leurs milieux d'appartenance pourrait constituer la poursuite d'un tel travail. Ceci permettrait de définir l'écart existant entre les croyances des enseignantes et les croyances des élèves qui semblent être particulièrement importantes pour ces dernières. 


\section{BIBLIOGRAPHIE}

CONNELL, R.W. (1995). Masculinities. Berkeley and Los Angeles : University of California Press.

CUSUMANO, D. L. \& THOMPSON, J. K. (1997). Body image and body shape ideals in magazines :

Exposure, awareness, and internalization. Sex Roles, 37, 701-722.

DESERTRAIN, G.S. \& WEISS, M.R. (1988). Being female and athletic : a cause for conflict ? Sex roles, 18, 567-582.

DURAND-DELVIGNE, A. (1990). Identité, catégorisation par sexes et pratiques pédagogiques. Revue internationale de psychologie sociale, 3/2,167-181.

FONTAYNE, P., SARRAZIN, P. \& FAMOSE, J.P. (2001). Les pratiques sportives des adolescents : une différenciation selon le genre. STAPS, 55, 23-38.

FOX, K.R. \& CORBIN, C.B. (1989). The Physical Self-Perception Profile : development and preliminary validation. Journal of sport and exercise psychology, 11, 408-430.

FRANZOI, S.L. (1995). The body-as-object versus the body-as-process : gender differences and gender considerations. Sex roles, 33, 417-437.

FREDRICKSON, B. L. \& ROBERTS, T. A. (1997). Objectification theory : toward understanding women's lived experiences and mental health risks. Psychology of Women Quarterly, 21, 173-206.

GREENLEAF, C. (2002). Athletic body image : exploratory interviews with former competitive female athlete. Women in sport \& physical activity journal, 11 (1), 63-71.

GROGAN, S. \& WAINWRIGHT, N. (1996). Growing up in the culture of slenderness. Girls experiences of body dissatisfaction. Women's studies international forum, 19, 665-673

KRANE, V. (2001). We can be athletic and feminine, but do we want to ? Challenging hegemonic femininity in women's sport. Quest, 53, 115-133

KRANE , V., CHOI, Y.L., BAIRD, M.S. \& KAUER, J.K. (2004). Living the paradox : female athlete negotiate femininity and muscularity. Sex roles, 50, 315-329.

MELVILLE, D.S. \& MADDALOZZO, J.G.F. (1988). The effects of a physical educator's appearance of body fatness on communicating exercise concepts to high school students. Journal of teaching in physical education, 7, 343-352.

MESSNER, M. A. (1988). Sports and male domination : the female athlete as contested ideological terrain. Sociology of sport journal, 5, 197-211.

MILLER, J.L. \& LEVY, G.D. (1996). Gender role conflict, gender-typed characteristic, self-concepts, and sport. Sex roles, 35, 111-122.

MUTH, J.L. \& CASH, T.F. (1997). Body-image attitudes : what differences does gender make? Journal of applied social psychology, 27, 1438-1452.

PUJADE-RENAUD, C. (1983). Le corps de l'enseignant dans la classe. Paris : ESF.

ROSS, S.R. \& SHINEW, K.J. (2007). Perspectives of women college athletes on sport and gender. Sex roles, 58, 40-57.

THEBERGE, N. (1985). Toward a feminist alternative to sport as male preserve. Quest, 37, 193-202. 
USSHER, J.M. (1997). Fantasies of femininity : Reframing the boundaries of sex. New Brunswick, NJ : Rutgers University Press.

WEBB, L. \& MACDONALD, D. (2007). Dualing with gender : teachers' work, careers and leadership in physical education. Gender and education, 19, 491-512.

WIENKE, C. (1998). Negociating the male body : men, masculinity, and cultural ideals. Journal of men's studies, 6 (3), 255.

WISEMAN, C.V., GRAY, J.J., MOSIMANN, J.E. \& AHRENS, A.H. (1992). Cultural expectation of thinness in woman : an update. International journal of eating disorders, 11, 85-89.

\section{NOTES}

1. S'il existe différents types de féminités et de masculinités, il semble de la même manière que les idéaux corporels soient pluriels. Mais, compte tenu de la place disponible, nous limiterons la présentation aux idéaux corporels précédemment cités.

\section{RÉSUMÉS}

Quelle est la place de la féminité dans l'image que les enseignantes d'EPS ont de leur corps au sein de la relation pédagogique et en dehors de celle-ci? L'EPS prenant appui sur les activités sportives, existe-t-il chez elles des tensions du fait de l'origine masculine du sport? Le but est de situer l'importance accordée aux idéaux corporels socialement valorisés, de caractériser l'image du corps d'enseignantes d'EPS, ainsi que les stratégies permettant de concilier image corporelle et idéal corporel.

What is the part which femininity plays in the PE female teachers' body image when teaching and in everyday life ? Sports activities are the foundation of PE : Do female PE teachers suffer tensions because of this masculine origin of PE? The aim is to point out the importance of social values attached to body image, the PE women teachers' body image, and the strategies of reconciling body image with body ideal.

\section{INDEX}

Keywords : body image, hegemonic femininity, ideal body, physical education

Mots-clés : EPS, féminité hégémonique, idéal corporel, image du corps

\section{AUTEURS \\ GENEVIÈVE COGÉRINO}

PU 


\section{MARIE MANSEY}

Professeur agrégée d'EPS, doctorante, Centre de Recherche sur l'Innovation en Sport (CRIS, EA 647), Université C. Bernard Lyon 1 